\title{
An Enhanced Approach to Determine A Small Forward Node Set Based on Multipoint Relays
}

\author{
Jie $\mathrm{Wu}$ \\ Dept. of Computer Science and Engineering \\ Florida Atlantic University \\ Boca Raton, Florida 33431 \\ Email: jie@cse.fau.edu
}

\begin{abstract}
Multipoint relays (MPR) [2] provide a localized and optimized way of determining a small forward node set for broadcasting messages in an ad hoc network. Using 2hop neighborhood information, each node determines a small set of forward neighbors to relay messages. Selected forward nodes form a connected dominating set (CDS) to ensure full coverage. Recently, Adjih, Jacquet, and Viennot [1] proposed a novel localized algorithm to construct a small forward node set based on MPR. In this paper, we provide a further extension to generate a smaller forward node set without additional cost. The effectiveness of our approach is confirmed through a simulation study.
\end{abstract}

\section{INTRODUCTION}

Wireless interfaces pose a unique challenge in designing efficient broadcasting in ad hoc networks. When a node sends a message, the message can reach all the adjacent nodes and, therefore, only a subset of nodes is needed to relay a broadcast message in ad hoc networks.

The efficient broadcasting in ad hoc networks can be formulated by identifying a small connected dominating set (CDS) in the network where nodes in the set and only nodes in the set relay the message. A set is dominating if every node in the network is either in the set or a neighbor of the set. Most existing works on finding a small CDS are not suitable for ad hoc networks, since they rely on either global information or global infrastructure. In an ad hoc network, network topology changes frequently and, hence, a global information/infrastructure approach may not be combinatorially stable. In a combinatorially stable system, the topology changes occur sufficiently slowly to allow successful propagation of all topology updates as necessary.

The localized approach is a solution in ad hoc networks to ensure the combinatorially stable property. In this approach, each node determines its status and/or the status of neighbors (forward or non-forward) based on neighborhood information (such as local topology). A generic broadcast scheme based on different ways of using neighborhood information is given in [5]. Multipoint relays (MPR) [2] is such a localized approach, where each forward node determines the status of its neighbors based on its 2-hop neighbor set through node coverage. Specifically, each forward node selects a subset of 1-hop neighbors to cover its 2-hop neighbor set. That is, each 2-hop neighbor is a neighbor of the selected subset of 1-hop neighbors.
The original MPR is source-dependent; that is, the forward node set is dependent on the source of the broadcast and communication latency. Recently, Adjih, Jacquet, and Viennot [1] proposed a novel source-independent MPR. The CDS is constructed based on MPR following two simple rules. In this paper, we enhance the source-independent MPR through a simple modification.

The rest of the paper is organized as follows. Section 2 provides preliminaries on general broadcasting in ad hoc networks and MPR and its extension. Section 3 proposes the enhanced MPR. Section 4 provides some simulation results and Section 5 concludes the paper.

\section{Preliminaries}

The simplist way to perform a broadcasting is based on the following rule.

- Blind flooding rule: a node re-transmits the message once and only once.

The blind flooding may cause excessive redundancy and results in channel contention and message collision (also called broadcast storm problem [3]). In Figure 1 (a), when node $u$ broadcasts, every other node relays once. In reality, either $w$ or $x$ is sufficient.

A dominating set (DS) is a subset of nodes in the network where every node is either in the subset or a neighbor of a node in the subset. A DS is called a connected dominating set (CDS) if the subgraph induced by the DS is connected. Limited broadcast relay is based on the following rule.

- CDS rule: a node re-transmits the message once and only once if it belongs to the CDS.

In Figure 1 (a), node $w$ forms a CDS and, hence, only $w$ forwards the message (except for the source). The problem is now reduced to finding a small CDS in a localized way.

\section{A. Multipoint Relays (MPR)}

Let $N(V)$ denote the set of all nodes that are in $V$ or have a neighbor in $V . V$ covers $U$ if $U \subset N(V)$. In multipoint relays (MPR) [2], each node $v$ maintains 2-hop neighbor set $N(N(v)$ ). Node $v$ selects a small forward node set, $C(v)$, from its 1-hop neighbor set to cover its 2-hop neighbor set; that is, $C(v) \cup v$ is a CDS for $N(N(v))$. C $(v)$ is also called the 
coverage set for $v$. When $u$ is selected by $v$ as a forward node, $v$ is called the selector of $u$. Note that several selectors may exist for a particular node. A forward node may or may not actually retransmit the message; its actual status is determined by the following MPR rule.

- MPR rule: a node re-transmits the message once and only once if the first message received is from a selector.

The collection of nodes that have re-transmitted the message plus the source node form a CDS.

Let $N_{1}(V)=N(V)-V$ denote the nodes at distance one from $V$ and $N_{2}(V)=N(N(V))-N(V)$ denote the nodes at distance two from $V$. A simple greedy algorithm for computing $C(v)$ (initially empty) at $v$ is as follows.

\section{Greedy Algorithm:}

- Add $u \in N_{1}(v)$ to $C(v)$, if there is a node in $N_{2}(v)$ covered only by $u$.

- Add $u \in N_{1}(v)$ to $C(v)$, if $u$ covers the largest number of nodes in $N_{2}(v)$ that have not been covered.

In Figure 1 (b), suppose the following coverage sets are selected based on the above greedy algorithm: $C(u)=\{v, y\}$, $C(v)=\{x\}, C(w)=\{y\}, C(x)=\{v\}$, and $C(y)=\{w\}$. Collectively nodes $v, w, x, y$ form a CDS. As specified in MPR, the actual set of forward nodes for a particular broadcast uses only a subset, and it depends on the location of the source and communication latency. For example, if $v$ is the source and node $x$ receives the first message from $v$, then $x$ is a forward node. Also, if nodes $w$ and $y$ receive their first message from $x$ and $v$, respectively, none of them will forward the message. Therefore, $\{v, x\}$ forms a CDS for this case. However, if node $y$ receives the first message from $u$, then $\{v, x, y\}$ forms a CDS.

\section{B. Source-independent MPR}

The original MPR is source-dependent. Adjih, Jacquet, and Viennot [1] recently proposed a novel localized algorithm to construct a CDS based on MPR, and it is source-independent. A node belongs to a CDS if

- Rule 1: the node has a smaller ID than all its neighbors.

- Rule 2: the node is a forward node selected by its neighbor with the smallest ID.

Applying Rule 1 and Rule 2 to Figure 1 (b), $\{x, y, v, u\}$ forms a CDS. Compared with the set derived from the original MPR, node $w$ is missing since it is selected by $y$ (which does not have the smallest ID among $w$ 's neighbors). In addition, node $u$ is included since it has a smaller ID than all its neighbors. The correctness of source-independent MPR is given in [1].

\section{EXTENSIONS}

We first observe two drawbacks in the source-independent MPR: (1) Rule 1 is "useless" in many occasions; that is, the node selected based on Rule 1 is not essential for a CDS. (2) The original MPR forward node selection does not take advantage of Rule 2.

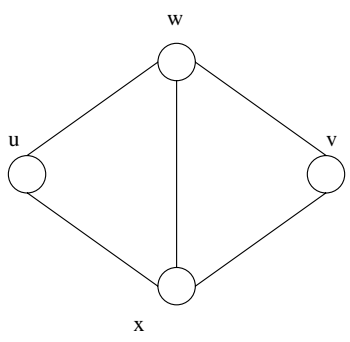

(a)

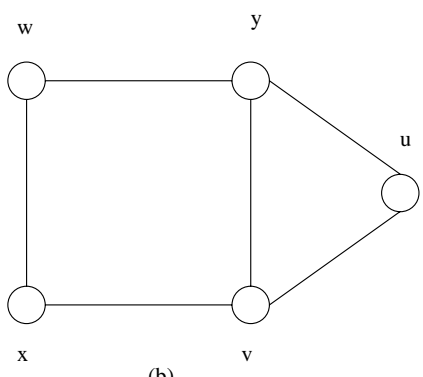

(b)

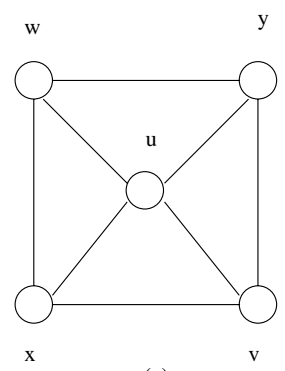

(c)

Fig. 1. Three sample networks.

In Figure 1 (a), $u$ and $v$ are selected based on Rule 1; however, they are useless. In fact, node $w$ alone is sufficient for a CDS. Similarly, $u$ selected by Rule 1 in Figure 1 (b) is useless. On the other hand, we might have to include some smallest ID nodes even if they are not selected by any of their neighbors as a forward node. In Figure 1 (c), suppose node $u$ is not selected by any of its neighbors, $u$ has to be included (as it is selected by Rule 1), because any forward node selected by a node other than $u$ will be ignored based on Rule 2 .

In Figure 1 (b), we assume that $v$ selects $x$ as its forward node. Based on Rule 2, since $v$ is the smallest ID neighbor of $x, x$ cannot ignore $v$ 's choice. On the other hand, if $v$ chooses $y$, since $v$ is not the smallest id neighbor of $y$, $v$ 's choice will be ignored by $y$. Therefore, forward node $y$ comes for "free" for $v$. That is, the inclusion of $y$ does not increase the size of the forward node set.

\section{A. Enhanced Rule 1}

In the following, we propose two extensions to sourceindependent MPR: one on Rule 1 and the other on the greedy algorithm.

- Enhanced Rule 1: the node has a smaller ID than all its neighbors and it has two unconnected neighbors. 
The Enhanced Rule 1 together with the original Rule 2 will generate a CDS under all cases except complete graphs. Note that when the network is complete, there is no need of CDS, because each source forms a CDS.

Theorem 1: If the given graph is not a complete graph, the set of forward nodes selected by the Enhanced Rule 1 and Rule 2 forms a CDS.

Proof: It has been shown in [1] that forward nodes selected by Rule 1 and Rule 2 form a CDS. We only need to show that whenever a smallest ID node $v$ within its 1-hop neighborhood is removed based on the Enhanced Rule 1, the resultant forward nodes still form a CDS.

Because the graph is not a complete graph and all of $v$ 's neighbors are pair-wise connected, there must exist a node that is not a neighbor of $v$. Let $w$ be such a node with the smallest ID. Since $v$ has the smallest ID in its 1-hop neighborhood, either $v$ or $w$ has the smallest ID in the 1-hop neighborhood of any neighbor of $v$. When one neighbor of $v$, say $u$, is selected by its smallest ID neighbor $v(w)$ to reach $w(v)$ in MPR, based on Rule 2, $u$ is a forward node and it covers $v$ and all neighbors of $v$. Therefore, $v$ can be removed.

\section{B. Enhanced forward node selection}

Node $u$ is a free neighbor of $v$ if $v$ is not the smallest ID neighbor of $u$. In the enhanced forward node selection, we first include all free neighbors, then nodes with higher degrees (i.e., covering more uncovered 2-hop neighbors) are selected and use node ID to break a tie if needed until $N_{2}(v)$ is covered.

\section{Extended Greedy Algorithm:}

- Add all free neighbors to $C(v)$.

- Add $u \in N_{1}(v)$ to $C(v)$, if there is an uncovered node in $N_{2}(v)$ covered only by $u$.

- Add $u \in N_{1}(v)$ to $C(v)$, if $u$ covers the largest number of uncovered nodes in $N_{2}(v)$ that have not been covered by the current $C(v)$. Use node ID to break a tie when two nodes cover the same number of uncovered nodes.

\section{Simulation}

A simulation study has been conducted to compare sourceindependent MPR (labeled MPR) with the enhanced MPR (labeled EMPR 1 with the Enhanced Rule 1 only, and EMPR $1 \& 2$ with the Enhanced Rule 1 and the enhanced forward node selection).

The simulation was conducted by randomly distributing a given number (range from 20 to 100 with a step of 10 and from 100 to 1,000 with a step of 100) of nodes in a $100 \times 100$ 2 -D space. Both dense graphs (Figure 2 with a transmission range of 18) and sparse graphs (Figure 3 with a transmission range of 6) have been simulated.

In both simulations, the enhanced MPR with both the Enhanced Rule 1 and the enhanced forward node selection has about $10 \%$ reduction of the forward node set compared with that of the source-independent MPR. The enhanced MPR with the Enhanced Rule 1 is more effective in relatively sparse

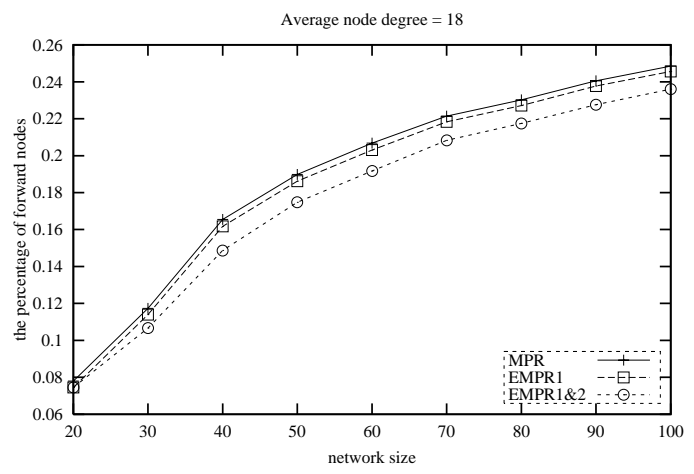

Average node degree $=18$

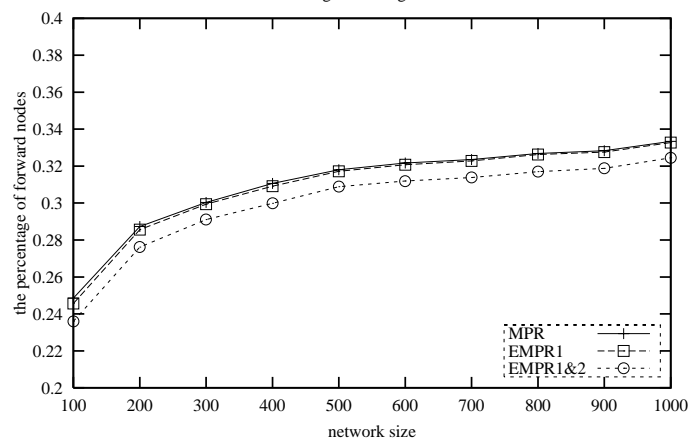

Fig. 2. Simulation results for dense graphs.

networks, whereas the enhanced MPR with enhanced forward node selection is more effective in relatively dense networks. This is because in a dense network, the probability of two unconnected neighbors increases as the number of neighbors increases (and the Enhanced Rule 1 is no longer effective). On the other hand, as the number of neighbors increases, the number of free neighbors also increases.

\section{CONCLUSions}

In this paper, we have proposed an enhanced sourceindependent MPR based on the recently proposed sourceindependent MPR. The enhancement is done without increasing the complexity of the method. The effectiveness of the enhancement is confirmed through a simulation study on both sparse and dense networks. In this paper, we did not consider energy-aware multiple relays selection. One straightforward extension is to use residue energy level as node priority instead of node ID. That is, the smallest ID node is replaced by the node with the highest residue energy level. In this case, a node with the highest residue energy in its 1-hop neighborhood has a better chance to become a forward node based on the Enhanced Rule 1. In this way, we can conduct an energy-aware broadcasting [4]

\section{REFERENCES}

[1] C. Adjih, P. Jacquet, and L. Viennot. Computing connected dominated sets with multipoint relays. Technical Report, INRIA, Oct. 2002.

[2] A. Qayyum, L. Viennot, and A. Laouiti. Multipoint relaying for flooding broadcast message in mobile wireless networks. Proc. HICSS-35, Jan. 2002 . 

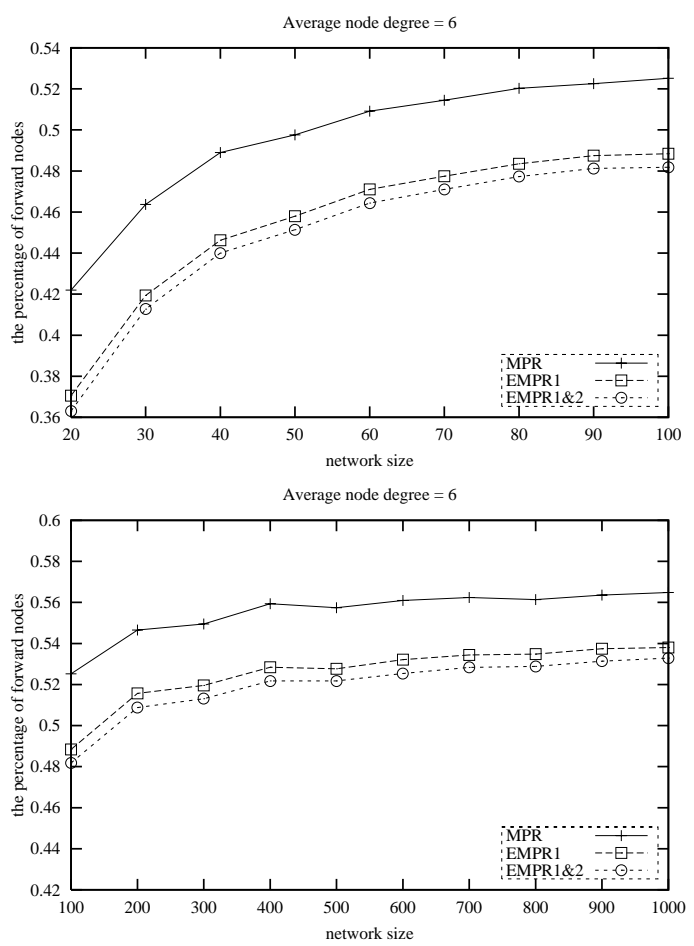

Fig. 3. Simulation results for sparse graphs.

[3] Y.-C. Tseng, S.-Y. Ni, Y.-S. Chen, and J.-P. Sheu. The broadcast storm problem in a mobile ad hoc network. Wireless Networks, 8(2/3):153-167, Mar.-May 2002.

[4] I. Stojmenovic, S. Seddigh, and J. Zunic, Dominating sets and neighbor elimination based broadcasting algorithms in wireless networks. IEEE Transactions on Parallel and Distributed Systems, 13(1):14-25, Jan. 2002.

[5] J. Wu and F. Dai. A generic distributed broadcast scheme in ad hoc wireless networks. Proc. of IEEE International Conference on Distributed Computing Systems, 460-467, May 2003. 